\title{
Three operational taxonomic units of Eimeria are common in Nigerian chickens and may undermine effective molecular diagnosis of coccidiosis
}

\author{
Isa D. Jatau ${ }^{1 *}$, Idris A. Lawal ${ }^{1}$, Jacob K. P. Kwaga', Fiona M. Tomley ${ }^{2}$, Damer P. Blake ${ }^{2^{*}}$ and Andrew J. Nok
}

\begin{abstract}
Background: Chicken is fast becoming the world's most consumed meat. As a consequence poultry health is more important now than ever before, with pathogens of chickens recognised as serious threats to food security. One such threat are Eimeria species parasites, protozoa which can cause the disease coccidiosis. Eimeria can compromise economic poultry production and chicken welfare, and have serious consequences for poor livestock keepers. Seven Eimeria species that infect chickens are recognised with a global enzootic distribution. More recently three cryptic Operational Taxonomic Units (OTUx, y and z) have been described in populations of Eimeria recovered from chickens in Australia. Two of the three OTUs have also been detected in sub-Saharan Africa, but their occurrence, pathology and the risk they pose is largely unknown.
\end{abstract}

Results: Nigeria has witnessed a dramatic expansion in poultry production and is now the largest poultry producer in Africa. Here, faecal samples collected from nine of 12 commercial chicken farms sampled in Kaduna state, Nigeria, were found to contain eimerian oocysts. After amplification by in vivo propagation all three cryptic OTU genotypes were detected using polymerase chain reaction (PCR), including OTUy for the first time outside of Australia. Comparison with a widely used, established Eimeria species-specific PCR assay revealed failure to detect the OTU genotypes.

Conclusions: All three of the Eimeria OTU genotypes appear to be common in north-western Nigeria. The failure of a leading species-specific molecular assay to detect these genotypes indicates a risk of false negative Eimeria diagnosis when using molecular tools and suggests that the spatial occurrence of each OTU may be far wider than has been recognised. The risk posed by these novel genotypes is unknown, but it is clear that a better understanding of Eimeria occurrence is required together with the validation of effective diagnostics.

Keywords: Eimeria, Chickens, Nigeria, Poultry, Prevalence, Operational taxonomic unit

\section{Background}

Poultry farming is one of the fastest growing sectors of animal production in the world with the greatest increases occurring in developing countries in Africa and Asia [1]. The expansion is predicted to continue for at least 30 years, posing enormous challenges for system development and pathogen control [2]. In

\footnotetext{
* Correspondence: mail4idjatau@gmail.com; idjatau@abu.edu.ng; dblake@rvc. ac.uk

${ }^{1}$ Faculty of Veterinary Medicine, Ahmadu Bello University, Zaria, Nigeria

${ }^{2}$ Pathology and Pathogen Biology, Royal Veterinary College, Hawkshead Lane, North Mymms AL9 7TA, UK
}

(c) 2016 The Author(s). Open Access This article is distributed under the terms of the Creative Commons Attribution 4.0 International License (http://creativecommons.org/licenses/by/4.0/), which permits unrestricted use, distribution, and reproduction in any medium, provided you give appropriate credit to the original author(s) and the source, provide a link to the Creative Commons license, and indicate if changes were made. The Creative Commons Public Domain Dedication waiver (http://creativecommons.org/publicdomain/zero/1.0/) applies to the data made available in this article, unless otherwise stated. small-scale poultry production systems for food, currency, work and business, but diseases such as coccidiosis are a major limiting factor $[3,4]$. Coccidiosis, caused by protozoan Eimeria species parasites, is a major recurring disease which exerts a negative effect on profitable and sustainable small-scale poultry enterprises around the world, including in Africa [5]. Seven recognized species of Eimeria specifically parasitize the digestive tract of domestic chickens (Gallus gallus domesticus) [6]. Eimeria acervulina, 
Eimeria maxima, Eimeria necatrix and Eimeria tenella are commonly considered to be most important as a consequence of their occurrence, fecundity and pathogenicity, but all species can undermine chicken health and welfare [2, 6, 7]. Concurrent infection with multiple Eimeria species is common and clinical manifestation of the disease can vary substantially [8]. Reports of three genetic variants (termed Eimeria operational taxonomic units or OTUs $\mathrm{x}, \mathrm{y}$ andz) circulating among poultry in Australia has added further complexity $[9,10]$, with two of these variants also detected recently in sub-Saharan Africa [5]. Morphological descriptions of OTU genotype oocysts are not currently available. Preliminary indications from limited genetic resources suggest OTUx and OTUy are most likely to be divergent strains of E. maxima and Eimeria brunetti respectively, while OTUz remains annotated as a cryptic species with its genetic relatedness to the seven recognised species unclear $[5,9]$ and [Clark et al., manuscript submitted].

Reports on the occurrence of Eimeria species in Nigerian chickens have previously been based on traditional protocols using morphological and pathological criteria in the diagnosis of coccidiosis $[11,12]$. While effective, these traditional methods can be subjective and unreliable in the diagnosis of natural infections, especially during sub-clinical infection when more than one species is present [13]. Consequentially, the use of molecular tools for accurate species identification and characterization of regional isolates of this parasite is valuable. Using molecular tools can reduce or obviate the requirement for time-consuming, and frequently subjective microscopic analysis, but relies on sequence conservation within the genomic regions targeted by the assays. The impact of genetic variation between isolates of the same species on PCR detection has been described previously for both E. maxima and Eimeria mitis, where multiple primer sets have been required for some targets [14]. The ability of many current molecular tools to identify parasites of the OTU genotypes is untested and may represent a risk of under-detection. PCR assays developed by Fornace and colleagues [5], and Godwin and Morgan [15] have attempted to address this deficit, although the latter requires equipment that is not currently widely available in many laboratories. Effective detection of circulating Eimeria species is important for proper diagnosis and disease control, and can inform the selection of appropriate anticoccidial drugs and vaccines [16, 17]. All seven of the recognised Eimeria species have been detected previously in Nigeria [12]. Here, we report the use of species-specific molecular assays to document the occurrence of all three Eimeria OTU genotypes in Nigeria with relevance to poultry husbandry and health.

\section{Results \\ Morphological identification of Eimeria species oocysts in commercial poultry}

Twelve farms found during previous routine veterinary monitoring to harbour coccidial oocysts were sampled. Oocysts were assigned putative species identity based upon microscopic morphology [18]. Briefly, small oocysts were categorised as E. acervulina and/or E. mitis (group AM, oocysts $\leq 18.8 \mu \mathrm{m}$ long), medium sized oocysts as E. necatrix, E. tenella and/or Eimeria praecox (group NTP, 18.9-23.8 $\mu \mathrm{m}$ long) and larger oocysts as $E$. brunetti and/or E. maxima (group BM, $\geq 23.9 \mu \mathrm{m}$ long). Nine samples were found to contain oocysts which were capable of sporulation, all of which contained medium sized oocysts consistent with the occurrence of NTP Eimeria species (75\% of the samples tested). Large oocysts, indicative of E. brunetti and/or E. maxima, were also observed in all nine oocyst positive samples (75 \%). Small oocysts, representing E. acervulina and/or $E$. mitis, were detected in eight samples (70 \%; Table 1).

Oocysts recovered from all nine positive samples were amplified by in vivo propagation because the original samples yielded low oocyst recovery and poor sporulation. Oocysts were harvested at post-mortem directly from the caeca to enrich the putative E. tenella subpopulation of each sample for related studies [19]. As a consequence, the species complexity was reduced in five of the nine samples (Table 1). Specifically, only four of eight previously positive samples retained detectable AM size oocysts, and four of nine samples retained BM sized oocysts. NTP sized oocysts persisted in all nine samples.

\section{Occurrence and identification of Eimeria using nested species-specific PCR}

Nested PCR targeting the seven recognised Eimeria species confirmed the occurrence of E. tenella in all NTP oocyst positive samples (Table 1). Eimeria praecox was not detected, but three of the nine samples were also found to contain E. necatrix. All four samples found to contain AM morphology-group oocysts were shown to include both E. acervulina and E. mitis genomic DNA, with two AM negative groups also found to contain $E$. mitis by nested PCR. In direct contrast, all four samples found to contain BM morphology-group oocysts were negative for E. brunetti and E. maxima genomic DNA by nested PCR (Table 1). Two amplicons representative of each species detected were cloned and sequenced (accession numbers LT549029-LT549036). BLASTn comparison against the GenBank non-redundant database confirmed species identity for each assay.

\section{Detection of Eimeria OTU genotypes by ITS-2 PCR}

All three OTU genotypes were detected by OTUspecific PCR targeting the internal transcribed spacer 
Table 1 Identification of Eimeria species and OTU genotypes recovered from commercial poultry farms in Zaria

\begin{tabular}{|c|c|c|c|c|c|c|c|c|c|c|c|c|c|c|c|c|}
\hline \multirow[b]{3}{*}{ Sample } & \multicolumn{16}{|c|}{ Oocyst morphology } \\
\hline & \multicolumn{3}{|c|}{ Faecal sample } & \multicolumn{3}{|c|}{ After passage } & \multicolumn{7}{|c|}{ Nested PCR } & \multicolumn{3}{|c|}{ OTU PCR } \\
\hline & $\mathrm{AM}$ & NTP & $\mathrm{BM}$ & $\mathrm{AM}$ & NTP & $\mathrm{BM}$ & $\mathrm{Ea}$ & Emi & Ene & $\mathrm{Et}$ & Ep & $\mathrm{Eb}$ & Ema & OTUx & OTUy & OTUz \\
\hline $1[\mathrm{~L}]$ & + & + & + & + & + & + & + & + & + & + & - & - & - & + & + & - \\
\hline $2[\mathrm{~B}]$ & + & + & + & + & + & + & + & + & + & + & - & - & - & + & + & + \\
\hline $3[\mathrm{~L}]$ & + & + & + & - & + & - & - & + & - & + & - & - & - & - & - & - \\
\hline $4[\mathrm{~B}]$ & + & + & + & - & + & + & - & - & - & + & - & - & - & - & - & - \\
\hline $5[\mathrm{~B}]$ & - & + & + & - & + & - & - & - & - & + & - & - & - & - & - & - \\
\hline 7 [L] & + & + & + & + & + & + & + & + & - & + & - & - & - & + & + & + \\
\hline 8 [B] & + & + & + & - & + & - & - & + & - & + & - & - & - & - & - & - \\
\hline $11[\mathrm{~B}]$ & + & + & + & - & + & - & - & - & - & + & - & - & - & - & + & - \\
\hline $12[\mathrm{~L}]$ & + & + & + & + & + & - & + & + & + & + & - & - & - & - & - & - \\
\hline Total & 8 & 9 & 9 & 4 & 9 & 4 & 4 & 6 & 3 & 9 & 0 & 0 & 0 & 3 & 4 & 2 \\
\hline
\end{tabular}

Eimeria occurrence was determined by oocyst morphology before and after in vivo propagation, and species/genotype-specific PCR after propagation. Highlighted boxes indicate samples found to contain large oocysts by microscopy after passage and OTUx and y genotypes, but not $E$. brunetti or $E$. maxima by PCR. Ea $=E$. acervulina, $\mathrm{Eb}=E$. brunetti, Ema $=E$. maxima, Emi $=E$. mitis, Ene $=E$. necatrix, Ep $=E$. praecox, Et $-E$. tenella. Morphological identification: $\mathrm{AM}=\mathrm{small}$ oocysts $($ Ea, $\mathrm{Em}), \mathrm{NTP}=$ medium oocysts (En, Et, Ep), BM= large oocysts (Eb, Ema). L = layer breed chicken (ISA Brown), B= broiler breed chicken (Cobb). $+=$ parasite detected, $-=$ parasite not detected

(ITS) 2 sequence from mixed populations of oocysts collected in Nigeria following in vivo passage and caecal harvest [5]. OTUy was most common, detected in four of nine samples screened (44.4\%). The OTUx genotype was detected in three of nine samples (33.3\%), and always in the presence of the OTUy genotype. All three OTUx and OTUy positive samples had previously been found to contain large (BM morphology-group) oocysts (Table 1). The OTUz genotype was detected in two of nine samples screened $(22.2 \%)$, both of which were also found to contain OTUx and OTUy genotypes. Two amplicons representative of each OTU genoptype were cloned and sequenced (accession numbers LT549037LT549042). BLASTn comparison against the GenBank non-redundant database confirmed genotype identity for each assay.

\section{Discussion}

The original objective of these studies was to recover $E$. tenella isolates from Nigerian poultry as a step towards population genetic characterisation [19]. For this reason samples were initially screened microscopically for the occurrence of oocysts of the NTP size and type (i.e. medium sized oocysts) [18]. The regional prevalence of each Eimeria species and genotype has not been calculated since the sample size was limited and the choice of farms sampled was biased, focusing on farms previously identified as coccidia positive. Nonetheless, the range of occurrence of each species was comparable to that described previously in the study area and in the southern part of Nigeria [12, 20, 21], possibly influenced by collection during the wet season when litter oocyst levels were likely to be elevated [22]. The reduction in Eimeria species complexity detected in many of the samples after in vivo propagation is likely to be a consequence of harvest by caecal, rather than faecal oocyst recovery 7 days post infection. The detection of Eimeria species not known to replicate in the caeca is likely to represent oocysts produced higher up the gastrointestinal track and transiting through the caeca at the time of sampling.

Eimeria of the OTUx and OTUz genotypes have previously been detected in sub-Saharan Africa, but until now the OTUy genotype has only been described in Australia $[5,9,10]$. Here, analysis of a small number of field isolates clearly indicated for the first time that all three OTU genotypes are circulating within Nigerian poultry. Four of nine samples tested were found to contain at least one OTU genotype (44\%), which is comparable to the occurrence detected within Australian flocks [10]. The risk posed by these genotypes is currently unclear. Limited evidence indicates that live vaccines such as Eimeriavax ${ }^{\circ} 4 \mathrm{M}$, formulated to include $E$. acervulina, E. maxima, E. necatrix and E. tenella, cannot prevent colonisation by OTU genotype parasites under field conditions [23]. It is likely that current anticoccidial prophylaxis will be capable of controlling these parasites, but it is equally likely that they will be capable of developing anticoccidial resistance as has been shown for other Eimeria/drug combinations [24]. The absence of severe disease associated specifically with OTU genotype parasites in Australia indicates that current control measures remain adequate, but as the range of anticoccidial drugs available is reduced, live and possible future subunit or recombinant vaccines may be compromised.

Comparison of microscopic observation of oocysts with PCR-based detection of Eimeria genomic DNA yielded variable results. NTP size-type oocysts were detected in all nine samples by microscopy and were confirmed by the detection of $E$. necatrix and/or $E$. 
tenella genomic DNA. Eimeria praecox genomic DNA was not detected and these parasites may not have been present, although this species reproduces in the duodenal loop with a prepatent period of just $84 \mathrm{~h}$ and may have been lost if present during caecal harvest occurring 7 days post infection [25]. AM size oocysts were detected in four of the nine samples tested following propagation through chickens. Molecular analysis confirmed the presence of E. acervulina and/or E. mitis genomic DNA in these samples and detected two further positive samples, possibly indicating greater sensitivity, although it should be noted that the PCR employed would have detected parasite genomic DNA from all lifecycle stages and would not have been restricted to the oocyst. The greatest inconsistency was detected for BM size-type oocysts. Oocyst detection indicated the presence of BM oocysts in four of the nine samples tested, but all were PCR negative for E. brunetti and E. maxima. Such variation between the microscopic and molecular approaches may have been associated with the limit of detection for the PCR assay, but the finding that three of the four BM oocyst positive samples contained genomic DNA of the OTUx and OTUy genotypes suggests that current molecular diagnostics are inadequate in regions where these parasites may be circulating. Thus, the true extent of the occurrence of OTU genotype Eimeria is unknown.

\section{Conclusions}

Application of molecular diagnostics for the occurrence of the seven recognised Eimeria species and OTU genotypes has detected all three OTUs circulating in Nigerian poultry. Inconsistencies between microscopic and molecular diagnostic approaches indicate that molecular tools require updating to include the OTUx, y and $\mathrm{z}$ genotypes. This is the first report of the OTUy genotype outside of Australia.

\section{Methods}

\section{Study area}

Kaduna state is among the seven states of the northwestern geopolitical zones of Nigeria. It is situated within the Sudan savannah vegetation zones of Nigeria with distinct dry and wet seasons. The dry season runs from October to April. The wet season begins in most parts of the state in May and lasts up to September or October, with mean annual rainfall of between 510$1140 \mathrm{~mm}$. The samples analysed here were collected from poultry units around Zaria, Nigeria, between April and July 2013.

\section{Farm selection and sample collection}

Combined faecal/litter samples representative of a pen, and thus several individual chickens, were collected from
12 commercial poultry farms found during previous routine veterinary monitoring to harbour coccidial oocysts. In each poultry house, samples were collected following an approximate $\mathrm{W}$-shaped path, starting and finishing in the corners of one of the long sides of the house [3]. Along this path, combined faecal/litter samples were collected manually, stopping every three strides to collect one handful which was placed in clean plastic bags and transported on ice pack to the Research Laboratory of the Department of Veterinary Parasitology and Entomology, Ahmadu Bello University-Zaria.

\section{Sample processing and morphometric oocyst identification}

In the laboratory, $200 \mathrm{~g}$ of each sample was weighed, transferred into a plastic beaker and soaked in approximately $500 \mathrm{ml}$ of distilled water overnight. The soaked samples were then homogenized by thorough stirring using a glass rod and filtered through a metal sieve (mesh size 300-500 $\mu \mathrm{m}$ ). The filtrate from each sample was allowed to sediment for one hour on the laboratory bench, after which the supernatant fluid was discarded into a clean beaker. The presence of oocysts was confirmed microscopically by transferring the equivalent of $10 \mathrm{ml}$ of sediment into a centrifuge tube and testing for the presence of coccidial oocysts using the saturated saline flotation technique described elsewhere [26]. Oocysts were assigned putative species identity based upon microscopic morphology [18]. For each positive sample, oocysts were recovered from the remaining sediment using the centrifugal flotation technique [26]. The harvested oocysts were re-suspended in distilled water and washed by centrifugation three to four times to remove the flotation solution (300 $\mathrm{g}$ for $5 \mathrm{~min}$ ). The sediment containing the oocysts was transferred into Petri-dishes, re-suspended in $2.5 \%(\mathrm{w} / \mathrm{v})$ potassium dichromate solution and allowed to sporulate at room temperature for 7 days with regular stirring.

After sporulation, oocysts within each sample were cleaned from the residual faecal debris by treatment with sodium hypochlorite ( $4 \%$ active chlorine) and three successive washes in distilled water as described elsewhere [27]. After cleaning, the oocysts were re-suspended in distilled water and enumerated using a modified-Fuchs Rosenthal counting chamber. The tubes containing the cleaned oocysts were clearly labelled with isolate number, date and amount of oocysts $/ \mathrm{ml}$, and stored at $4{ }^{\circ} \mathrm{C}$ until required.

\section{Eimeria propagation}

Field samples found to contain coccidial oocysts which had sporulated were used for in vivo propagation as a consequence of overall low oocyst recovery and poor sporulation. Individually caged 4 week old specific- 
pathogen free (SPF) Light Sussex chickens were inoculated orally with 4,000 sporulated mixed oocysts from single field parasite populations. Progeny oocysts were recovered from caecal tissue and contents collected during post-mortem 7 days post infection, sporulated and purified as described elsewhere [25, 27].

\section{Total genomic DNA extraction}

Four millilitres of each washed oocyst suspension, containing between 2 and 5 million oocysts after in vivo propagation, were centrifuged $(750 \mathrm{~g}$ for $10 \mathrm{~min}$ ) to pellet the oocysts. Each pellet was re-suspended in the minimum volume residual supernatant and transferred to a $1.5 \mathrm{ml}$ screw top plastic tube. Glass beads (0.4-0.6 mm; Sigma, UK) equivalent to the volume of the oocyst pellet were added to the tube and covered with sterile phosphate buffered saline (PBS; pH 8.0). The pelleted oocysts were then disrupted using a Mini Beadbeater-8, (Biospec Products, Bartlesville, USA) for two minutes and total genomic DNA (gDNA) was isolated from the smashed oocyst homogenate using a QIAamp DNA Tissue mini kit (Qiagen, Germany) following the manufacturers protocol.

\section{Molecular identification of Eimeria by nested polymerase chain reaction}

A standardized nested PCR assay targeting the internal transcribed spacer (ITS)-1 sequence for identification of Eimeria species of poultry was used to improve detection of minority Eimeria species populations. Primers amplifying the entire ITS-1 sequence based in the flanking $18 \mathrm{~S}$ and 5.8S rDNA regions of the eimerian genome were used in the first genus-specific PCR phase, while speciesspecific primers targeting the ITS- 1 region were used to amplify the individual Eimeria species in the second nested phase. The primers (as shown in Additional file 1) and the PCR conditions used were as described previously $[3,14]$. Genomic DNA purified from the Houghton reference strains of each of the seven recognised Eimeria species were used as positive controls, with molecular grade water (Sigma, UK) used as the negative control starting from the beginning of the nested assay. The amplification products of the specific nested PCR were analysed by gel electrophoresis in $2 \%(\mathrm{w} / \mathrm{v})$ agarose gels in $1 \mathrm{x}$ Tris Acetate EDTA (TAE; all Sigma, UK) buffer stained with $0.01 \%$ (v/v) SafeView nucleic acid dye (NBS Biologicals, UK).

\section{Molecular identification of new operational taxonomic unit (OTU) cryptic Eimeria genotypes}

Primers specific to the OTUx, OTUy and OTUz ITS-2 sequences were used to screen each sample for the occurrence of these novel genotypes (Additional file 1). PCR conditions were as described previously and the PCR products were analysed by gel electrophoresis as described above [5].
PCR amplicon sequencing to confirm and validate species/genotype identification

Two PCR fragments representative of each Eimeria species detected were sequenced to confirm amplicon identity and validate PCR detection, resulting in 14 sequences from 31 positive reactions (45\%). Amplicons were purified using a Qiagen PCR purification kit, cloned using pGEM-T Easy (Promega, Madison, USA) in XL1-Blue MRF Escherichia coli (Stratagene, La Jolla, USA), miniprepped (Qiagen) and sequenced (GATC Biotech, Konstanz, Germany) as described by the respective manufacturers. Sequence assembly, annotation and interrogation were undertaken using CLC Main Workbench v6.0.2 (CLC Bio, Katrinebjerg, Denmark) and sequences were identified using BLASTn against the GenBank non-redundant database with default parameters. The sequences have been submitted to GenBank under the accession numbers LT549029-LT549042.

\section{Additional file}

Additional file 1: Polymerase chain reaction ( $P C R$ ) primers used for molecular identification of Eimeria species and OTU genotypes. (DOCX $23 \mathrm{~kb}$ )

\section{Acknowledgements}

The work described here was funded in part by the UK Department for International Development (DFID) and the Biotechnology and Biological Sciences Research Council (BBSRC) through the Combating Infectious Diseases of Livestock for International Development (CIDLID) initiative under project reference BB/H009337. This manuscript has been assigned the reference number PPB_01103 by the Royal Veterinary College.

\section{Authors' contributions}

IDJ, IAL, JKPK and AJN designed the sampling strategy and collected the samples. IDJ led the experimental work with input from DPB. IDJ and DPB drafted the manuscript with contributions from IAL, JKPK, FMT and AJN.

All authors read and approved the final manuscript.

Competing interests

The authors declare that they have no competing interests.

Consent for publication

Not applicable.

\section{Ethics approval and consent to participate}

This study was carried out in strict accordance with the Animals (Scientific Procedures) Act 1986, an Act of Parliament of the United Kingdom.

All animal studies and protocols were approved by the Royal Veterinary College ethics committee and the United Kingdom Government Home Office under the project licence 70/7781. Field samples were imported into the UK under Importation of Animal Pathogens Order (IAPO) permit PATH/71/2011/13 issued by the Department for Environment, Food and Rural Affairs.

Received: 6 October 2015 Accepted: 30 May 2016

Published online: 04 June 2016

\section{References}

1. Grace D, Mutua F, Ochungo P, Kruska R, Jones K, Brierley L, Lapar L, Said M, Herrero M, Phuc PD et al: Mapping of poverty and likely zoonoses hotspots. In.: Department for International Development, UK; 2012.

2. Blake DP, Tomley FM. Securing poultry production from the ever-present Eimeria challenge. Trends Parasitol. 2014;30(1):12-9. 
3. Kumar S, Garg R, Moftah A, Clark EL, Macdonald SE, Chaudhry AS, Sparagano O, Banerjee PS, Kundu K, Tomley FM, et al. An optimised protocol for molecular identification of Eimeria from chickens. Vet Parasitol. 2014;199(1-2):24-31.

4. Perry B, Randolph T, McDermott J, Sones K, Thornton P. Investing in animal health research to alleviate poverty. Nairobi, Kenya: ILRI (International Livestock Research Institute); 2002.

5. Fornace KM, Clark EL, Macdonald SE, Namangala B, Karimuribo E, Awuni JA, Thieme O, Blake DP, Rushton J. Occurrence of Eimeria species parasites on small-scale commercial chicken farms in Africa and indication of economic profitability. PLOS ONE. 2013;8(12):e84254.

6. Shirley MW, Smith AL, Tomley FM. The biology of avian Eimeria with an emphasis on their control by vaccination. Adv Parasitol. 2005;60:285-330.

7. Williams RB, Marshall RN, Pages M, Dardi M, del Cacho E. Pathogenesis of Eimeria praecox in chickens: virulence of field strains compared with laboratory strains of E. praecox and Eimeria acervulina. Avian Pathol. 2009; 38(5):359-66.

8. Haug A, Gjevre AG, Skjerve E, Kaldhusdal M. A survey of the economic impact of subclinical Eimeria infections in broiler chickens in Norway. Avian Pathol. 2008;37(3):333-41.

9. Cantacessi C, Riddell S, Morris GM, Doran T, Woods WG, Otranto D, Gasser RB. Genetic characterization of three unique operational taxonomic units of Eimeria from chickens in Australia based on nuclear spacer ribosomal DNA. Vet Parasitol. 2008;152(3-4):226-34.

10. Godwin RM, Morgan JA. A molecular survey of Eimeria in chickens across Australia. Vet Parasitol. 2015;214(1-2):16-21.

11. Muazu A, Masdooq A, Ngbede J, Salihu A, Haruna G, Habu A, Sati M, Jamilu $\mathrm{H}$. Prevalence and identification of species of Eimeria causing coccidiosis in poultry within Vom, Plateau State, Nigeria. Int J Poult Sci. 2008;7:917-8.

12. Jatau I, Sulaiman N, Musa I, Lawal A, Okubanjo O, Isah I, Magaji Y. Prevalence of coccidia infection and preponderance Eimeria species in free range indigenous and intensively managed exotic chickens during hot - wet season, in Zaria, Nigeria. Asian J Poult Sci. 2012;6:79-88.

13. Woods WG, Whithear KG, Richards DG, Anderson GR, Jorgensen WK, Gasser RB. Single-strand restriction fragment length polymorphism analysis of the second internal transcribed spacer (ribosomal DNA) for six species of Eimeria from chickens in Australia. Int J Parasitol. 2000;30(9):1019-23.

14. Lew $A E$, Anderson GR, Minchin CM, Jeston PJ, Jorgensen WK. Inter- and intra-strain variation and PCR detection of the internal transcribed spacer 1 (ITS-1) sequences of Australian isolates of Eimeria species from chickens. Vet Parasitol. 2003;112(1-2):33-50.

15. Godwin RM, Morgan JA. A simple, one-tube assay for the simultaneous detection and diagnosis of ten Australian poultry Eimeria. Electrophoresis. 2014;35(4):494-502.

16. Sun XM, Pang W, Jia T, Yan WC, He G, Hao LL, Bentue M, Suo X. Prevalence of Eimeria species in broilers with subclinical signs from fifty farms. Avian Dis. 2009:53(2):301-5.

17. Lee BH, Kim WH, Jeong J, Yoo J, Kwon YK, Jung BY, Kwon JH, Lillehoj HS, Min W. Prevalence and cross-immunity of Eimeria species on Korean chicken farms. J Vet Med Sci. 2010;72(8):985-9.

18. Haug A, Gjevre AG, Thebo P, Mattsson JG, Kaldhusdal M. Coccidial infections in commercial broilers: epidemiological aspects and comparison of Eimeria species identification by morphometric and polymerase chain reaction techniques. Avian Pathol. 2008;37(2):161-70.

19. Blake D, Clark E, Macdonald S, Thenmozhi V, Kundu K, Garg R, Jatau I, Ayoade S, Kawahara F, Moftah A, et al. Population, genetic and antigenic diversity of the apicomplexan Eimeria tenella and their relevance to vaccine development. Proc Natl Acad Sci U S A. 2015;112:E5343-50.

20. Etuk E, Okoli I, Uko M. Prevalence and Management Issues Associated with Poultry Coccidiosis in Abak Agricultural Zone of Akwa Ibom State, Nigeria. Int J Poult Sci. 2004;3:135-9.

21. Adene D, Oluleye O. Coccidiosis of poultry: The biology diagnosis, treatment and control. In: Poultry Health and Production Principles and Practices. Ibadan, Nigeria: Stirling-Horden Publishers (Nig.) Ltd 2004. p. 129-64.

22. Mungube EO, Bauni SM, Tenhagen BA, Wamae LW, Nzioka SM, Muhammed $L$, Nginyi JM. Prevalence of parasites of the local scavenging chickens in a selected semi-arid zone of Eastern Kenya. Trop Anim Health Prod. 2008; 40(2):101-9.
23. Morris GM, Woods WG, Richards DG, Gasser RB. Investigating a persistent coccidiosis problem on a commercial broiler-breeder farm utilising PCR-coupled capillary electrophoresis. Parasitol Res. 2007;101(3):583-9.

24. Chapman H. Biochemical, genetic and applied aspects of drug resistance in Eimeria parasites of the fowl. Avian Pathol. 1997;26:221-44.

25. Long $P$, Joyner L, Millard B, Norton C. A guide to laboratory techniques used in the study and diagnosis of avian coccidiosis. Folia Vet Lat. 1976;6(3):201-17.

26. Soulsby EJ: Helminths, Protozoa and Arthropods of Domesticated Animals, 7th Edition edn: ELBS, London Baillaire and Tindall; 1986.

27. Eckert J, Braun R, Shirley M, Coudert P. Guidelines on techniques in coccidiosis research. Brussels, Luxembourg: European Commission; 1995

\section{Submit your next manuscript to BioMed Central and we will help you at every step:}

- We accept pre-submission inquiries

- Our selector tool helps you to find the most relevant journal

- We provide round the clock customer support

- Convenient online submission

- Thorough peer review

- Inclusion in PubMed and all major indexing services

- Maximum visibility for your research

Submit your manuscript at www.biomedcentral.com/submit
) Biomed Central 